PROCEEDINGS OF THE

AMERICAN MATHEMATICAL SOCIETY

Volume 136, Number 12, December 2008, Pages 4339-4347

S 0002-9939(08)09402-1

Article electronically published on June 3, 2008

\title{
NUMERICAL PEAK POINTS AND NUMERICAL ŠILOV BOUNDARY FOR HOLOMORPHIC FUNCTIONS
}

\author{
SUNG GUEN KIM
}

(Communicated by N. Tomczak-Jaegermann)

\begin{abstract}
In this paper, we characterize the numerical and numerical strongpeak points for $\mathcal{A}_{\infty}\left(B_{E}: E\right)$ when $E$ is the complex space $l_{1}$ or $C(K)$. We also prove that $\left\{\left(x, x^{*}\right) \in \Pi\left(l_{1}\right):\left|x^{*}\left(e_{n}\right)\right|=1\right.$ for all $\left.n \in \mathbb{N}\right\}$ is the numerical Šilov boundary for $\mathcal{A}_{\infty}\left(B_{l_{1}}: l_{1}\right)$.
\end{abstract}

\section{INTRODUCTION}

Throughout this paper we will just consider complex Banach spaces. For a Banach space $E, S_{E}$ and $B_{E}$ will be the unit sphere and the closed unit ball of $E$, respectively. If $E$ and $F$ are Banach spaces, $\mathcal{C}_{b}\left(B_{E}: F\right)$ denotes the Banach space of the bounded continuous functions $f: B_{E} \rightarrow F$, endowed with the supremum norm. In the case that $F=\mathbb{C}$, we write $\mathcal{C}_{b}\left(B_{E}\right)$. An $N$-homogeneous polynomial $P$ from $E$ to $F$ is a mapping such that there is an $N$-linear (and bounded) mapping $L$ from $E$ to $F$ satisfying

$$
P(x)=L(x, \ldots, x), \quad \forall x \in E .
$$

The set of all $N$-homogenous polynomials from $E$ to $F$ is denoted by $\mathcal{P}\left({ }^{N} E: F\right)$. We denote by $\mathcal{A}_{\infty}\left(B_{E}: F\right)$ the Banach space of the bounded continuous function $f: B_{E} \rightarrow F$ such that $f$ is holomorphic on the open unit ball, endowed with the supremum norm. If $F=\mathbb{C}$, we write $\mathcal{A}_{\infty}\left(B_{E}\right)$.

A result of Šilov asserts that if $\mathcal{A}$ is a unital separating subalgebra of $C(K)(K$ is a compact Hausdorff topological space), there is a smallest closed subset $S \subset K$ such that every function of $\mathcal{A}$ attains its norm at some point of $S$ ([7], Theorem I.4.2). Bishop [5] proved that if $K$ is metrizable, in fact, there is a minimal subset of $K$ satisfying the above condition for every separating subalgebra of $C(K)$. That subset is the set of peak points for $\mathcal{A}$. Globevnik [8] introduced the corresponding concepts of the boundary of a subalgebra $\mathcal{A}$ of $\mathcal{C}_{b}(\Omega)$, the set of bounded and continuous functions on a topological space $\Omega$ not necessarily compact, and studied

Received by the editors September 9, 2006, and, in revised form, July 16, 2007, October 18, 2007, and October 23, 2007.

2000 Mathematics Subject Classification. Primary 46A22; Secondary 46G25.

Key words and phrases. Numerical peak points, numerical Silov boundaries.

The author thanks the referee for invaluable suggestions and for help with an earlier version of this paper.

(C)2008 American Mathematical Society Reverts to public domain 28 years from publication 
the boundaries for $\Omega=B_{c_{0}}$ and $\mathcal{A}$ a certain space of holomorphic functions. A subset $B \subset B_{E}$ is a boundary for a subspace $\mathcal{A} \subset \mathcal{C}_{b}\left(B_{E}\right)$ if

$$
\|f\|=\sup _{z \in B}|f(z)|, \quad \forall f \in \mathcal{A} .
$$

An element $x \in S_{E}$ is called a peak point for $\mathcal{A}$ if there exists $h \in \mathcal{A}$ such that $|h(x)|=\|h\|_{B_{E}}$ and $|h(z)|<\|h\|_{B_{E}}$ for every $z \in B_{E} \backslash\{x\}$. In this case we say that $h$ peaks at $x$. A peak point $x$ is called a strong peak point for $\mathcal{A}$ if there exists $h \in \mathcal{A}$ such that $|h(x)|=\|h\|_{B_{E}}$ and, given $\epsilon>0$, there exists a $\delta>0$ such that for every $z \in B_{E}$ with $\|z-x\|>\epsilon$, we have $|h(z)|<\|h\|_{B_{E}}-\delta$. In this case we say that $h$ peaks strongly at $x$.

In 1971, Harris [9] introduced the definition of a spatial numerical range for a bounded and holomorphic function defined on a Banach space and the corresponding concept of numerical radius. The spatial numerical range of a bounded function $f$ from $B_{E}$ to $E$ is given by

$$
W(f):=\left\{x^{*}(f(x)):\left(x, x^{*}\right) \in \Pi(E)\right\},
$$

where we denoted by $\Pi(E)$ the following subset:

$$
\Pi(E):=\left\{\left(x, x^{*}\right) \in S_{E} \times S_{E^{*}}: x^{*}(x)=1\right\} .
$$

The numerical radius $v(f)$ is just the number

$$
v(f):=\sup \{|\lambda|: \lambda \in W(f)\} .
$$

For more background and information about numerical ranges and radii, we refer the reader to [6]. For a linear space $\mathcal{A} \subset \mathcal{C}_{b}\left(B_{E}: E\right)$, M. Acosta and the author [3] gave the corresponding definition of numerical boundary for $\mathcal{A}$. We say that $B \subset \Pi(E)$ is called a numerical boundary for $\mathcal{A}$ if

$$
\sup _{\left(x, x^{*}\right) \in B}\left|x^{*}(f(x))\right|=v(f), \quad \forall f \in \mathcal{A} .
$$

In the case that $B$ is a $\left(\|\| \times w^{*}\right)$-closed numerical boundary for $\mathcal{A}$ that is minimal under the previous conditions, $B$ is said to be the numerical Silov boundary. In [3], the authors studied numerical boundaries for holomorphic functions on some classical Banach spaces. Parallel to the concepts of peak and strong peak-points, we introduce the corresponding definition of numerical and numerical strong-peak points. An element $\left(x, x^{*}\right) \in \Pi(E)$ is called a numerical peak point for $\mathcal{A}$ if there exists $h \in \mathcal{A}$ such that $\left|x^{*}(h(x))\right|=v(h)$ and $\left|z^{*}(h(z))\right|<v(h)$ for every $\left(z, z^{*}\right) \in \Pi(E) \backslash\left\{\left(x, x^{*}\right)\right\}$. In this case we say that $h$ peaks numerically at $\left(x, x^{*}\right)$. Also, $\left(x, x^{*}\right) \in \Pi(E)$ is called a numerical strong-peak point for $\mathcal{A}$ if there exists a function $h \in \mathcal{A}$ such that $\left|x^{*}(h(x))\right|=v(h)$, and for any $\left(x_{n}, x_{n}^{*}\right) \in \Pi(E)$ with $\lim _{n \rightarrow \infty}\left|x_{n}^{*}\left(h\left(x_{n}\right)\right)\right|=v(h)$ we have that $x_{n} \rightarrow x$ in norm and $x_{n}^{*} \rightarrow x^{*}$ in $\mathrm{w}^{*}$-topology. In this case we say that $h$ peaks strong-numerically at $\left(x, x^{*}\right)$. It is clear that every numerical strong-peak point is a numerical peak point. Note that if $E$ is a finite dimensional space, then every numerical peak point is also a numerical strong-peak point. It is immediate from definition that every $\left(\|\| \times w^{*}\right)$-closed numerical boundary for $\mathcal{A}_{\infty}\left(B_{E}: E\right)$ contains all numerical strong-peak points.

In Section 2, we characterize the numerical peak points for $\mathcal{A}_{\infty}\left(B_{l_{1}}: l_{1}\right)$ and prove that every numerical peak point for $\mathcal{A}_{\infty}\left(B_{l_{1}}: l_{1}\right)$ is also a numerical strongpeak point. We prove that $\left\{\left(x, x^{*}\right) \in \Pi\left(l_{1}\right):\left|x^{*}\left(e_{n}\right)\right|=1\right.$ for all $\left.n \in \mathbb{N}\right\}$ is the numerical Šilov boundary for $\mathcal{A}_{\infty}\left(B_{l_{1}}: l_{1}\right)$. 
In Section 3, we characterize the numerical peak points for $\mathcal{A}_{\infty}\left(B_{C(K)}: C(K)\right)$ when $K$ is a compact metrizable space. If $K$ is an infinite compact Hausdorff topological space, we prove that there are no numerical strong-peak points for $\mathcal{A}_{\infty}\left(B_{C(K)}: C(K)\right)$.

\section{Numerical PEAK POINTS AND NUMERICAL ŠILOV BOUndARY FOR HOLOMORPHIC FUNCTIONS ON $l_{1}$}

Lemma 2.1. If $\left(x, x^{*}\right) \in \Pi\left(l_{1}\right)$ is a numerical peak point for $\mathcal{A}_{\infty}\left(B_{l_{1}}: l_{1}\right)$, then $\left|x^{*}\left(e_{n}\right)\right|=1$ for all $n \in \mathbb{N}$.

Proof. Let $x:=\left(v_{n}\right)_{n} \in S_{l_{1}}, x^{*}:=\left(w_{n}\right)_{n} \in S_{l_{\infty}}$. Assume that there exists a positive integer $n_{0}$ such that $\left|w_{n_{0}}\right|<1$. Since the subset of peak points in $S_{E}$ for $\mathcal{A}_{\infty}\left(B_{l_{1}}\right)$ is invariant under surjective linear isometries on $l_{1}$, we may assume that $n_{0}=1$, so $\left|w_{1}\right|<1$. Let $h \in \mathcal{A}_{\infty}\left(B_{l_{1}}: l_{1}\right)$ peak numerically at $\left(x, x^{*}\right)$ with $h:=\left(h_{n}\right)_{n}$ for some $h_{n} \in \mathcal{A}_{\infty}\left(B_{l_{1}}\right)$. Let $x_{\lambda}^{*}:=\lambda e_{1}+\sum_{n>1} w_{n} e_{n}$ for $|\lambda| \leq 1$. We claim that $v_{1} \neq 0$. If $v_{1}=0$, then $\left(x, x_{\lambda}^{*}\right) \in \Pi\left(l_{1}\right)$ for $|\lambda| \leq 1$. We define the polynomial $\psi: \bar{D}(0,1) \rightarrow \mathbb{C}$ by

$$
\psi(\lambda):=\lambda h_{1}(x)+\sum_{n>1} w_{n} h_{n}(x)(|\lambda| \leq 1) .
$$

Since $\max _{|\lambda| \leq 1}|\psi(\lambda)|=v(h)=\left|x^{*}(h(x))\right|=\left|\psi\left(w_{1}\right)\right|$, by the Maximum Modulus Theorem, $\psi(\bar{\lambda})=v(h)$ for all $|\lambda| \leq 1$. Choose any complex number $\beta$ with $|\beta|=1$. Then $\left(x, x_{\beta}^{*}\right) \neq\left(x, x^{*}\right) \in \Pi\left(l_{1}\right)$ and $|\psi(\beta)|=\left|x_{\beta}^{*}(h(x))\right|=v(h)$. Since $h$ peaks numerically at $\left(x, x^{*}\right)$, we have a contradiction. Thus $v_{1} \neq 0$. We define the 1 degree polynomial $u: \bar{D}(0,1) \rightarrow \mathbb{C}$ by

$$
u(\lambda):=\lambda v_{1}+\sum_{n>1} w_{n} v_{n}(|\lambda| \leq 1) .
$$

Since $1=x^{*}(x)=\left|u\left(w_{1}\right)\right|$, by the Maximum Modulus Theorem, $u(\lambda)=1$ for all $|\lambda| \leq 1$. By the same reason as in the above argument, $\left(x, x_{\beta}^{*}\right) \in \Pi\left(l_{1}\right)$ and $|\psi(\beta)|=$ $\left|x_{\beta}^{*}(h(x))\right|=v(h)$ for any $\beta \in \mathbb{C}$ with $|\beta|=1$. Thus we have a contradiction. Therefore $\left|w_{1}\right|=1$.

Theorem 2.2. $S:=\left\{\left(x, x^{*}\right) \in \Pi\left(l_{1}\right):\left|x^{*}\left(e_{n}\right)\right|=1\right.$ for all $\left.n \in \mathbb{N}\right\}$ is the set of all numerical strong-peak points for the space of 2-degree polynomials in $\mathcal{A}_{\infty}\left(B_{l_{1}}: l_{1}\right)$ and every numerical peak point for the space of 2 -degree polynomials in $\mathcal{A}_{\infty}\left(B_{l_{1}}: l_{1}\right)$ is a numerical strong-peak point.

Proof. By Lemma 2.1, it is enough to show the first statement of the theorem. Let $\left(y_{0}, y_{0}^{*}\right) \in S$. We will prove that $\left(y_{0}, y_{0}^{*}\right)$ is a numerical strong-peak point for the space of 2 -degree polynomials in $\mathcal{A}_{\infty}\left(B_{l_{1}}: l_{1}\right)$. Let $J=\operatorname{supp}\left(y_{0}\right)$. Since the subset of peak points in $S_{E}$ for $\mathcal{A}_{\infty}\left(B_{l_{1}}\right)$ is invariant under surjective linear isometries on $l_{1}$, we can assume that $y_{0}(k)>0$ for all $k \in J$. Let $a_{n}:=y_{0}^{*}\left(e_{n}\right)$ for all $n \in \mathbb{N}$. Then $a_{k}=1$ for all $k \in J$. By Theorem 2.6 in [4] there exists a 2-degree polynomial $f \in \mathcal{A}_{\infty}\left(B_{l_{1}}\right)$ which peaks strongly at $y_{0}$ with $\|f\|=1$. Let $\lambda_{n}:=\operatorname{sign}\left(a_{n}\right)$ and $z_{0}:=\sum_{n=1}^{\infty} \lambda_{n} b_{n} e_{n} \in S_{l_{1}}$ with $b_{n}>0$ for all $n \in \mathbb{N}$. We define a 2-degree polynomial $h \in \mathcal{A}_{\infty}\left(B_{l_{1}}: l_{1}\right)$ by $h(x):=f(x) z_{0}\left(x \in l_{1}\right)$. Clearly $y_{0}^{*}\left(h\left(y_{0}\right)\right)=1=\|h\|=v(h)$. We claim that $h$ peaks strong-numerically at $\left(y_{0}, y_{0}^{*}\right)$. Consider a sequence $\left\{\left(x_{n}, x_{n}^{*}\right)\right\}$ in $\Pi\left(l_{1}\right)$ such that $\lim _{n \rightarrow \infty}\left|x_{n}^{*}\left(h\left(x_{n}\right)\right)\right|=v(h)$. We 
will prove that $x_{n} \rightarrow y_{0}$ in norm and $x_{n}^{*} \rightarrow y_{0}^{*}$ in $\mathrm{w}^{*}$-topology. Since

$$
1=\lim _{n \rightarrow \infty}\left|x_{n}^{*}\left(h\left(x_{n}\right)\right)\right| \leq \lim _{n \rightarrow \infty}\left|f\left(x_{n}\right)\right| \leq 1
$$

and $f$ peaks strongly at $y_{0}, x_{n} \rightarrow y_{0}$ in norm. Write $x_{n}^{*}:=\left(a_{j}^{(n)}\right)_{j} \in S_{l_{\infty}}$ and $x_{n}:=\left(c_{j}^{(n)}\right)_{j} \in S_{l_{1}}$ for all $n, j \in \mathbb{N}$. Let $x:=\left(d_{j}\right) \in l_{1}$. First we claim that $\lim _{n \rightarrow \infty} a_{k}^{(n)}=1$ for all $k \in J$. Let $i_{0} \in J$ be fixed. Let $\left\{a_{i_{0}}^{\left(n_{i}\right)}\right\}$ be any subsequence of $\left\{a_{i_{0}}^{(n)}\right\}$. As the set $\left\{a_{i_{0}}^{\left(n_{i}\right)}\right\}$ is a bounded subset of $\mathbb{C}$, there exist a subsequence $\left\{a_{i_{0}}^{\left(n_{i_{l}}\right)}\right\}$ and a complex number $r$ with $|r| \leq 1$ such that $\lim _{l \rightarrow \infty} a_{i_{0}}^{\left(n_{i_{l}}\right)}=r$. Since $1=x_{n}^{*}\left(x_{n}\right)$ for all $n \in \mathbb{N}$, we have

$$
1=\sum_{j=1}^{\infty} a_{j}^{\left(n_{i_{l}}\right)} c_{j}^{\left(n_{i_{l}}\right)} \text { for all } l \in \mathbb{N} .
$$

Since $x_{n} \rightarrow y_{0}$ in norm, we have $\lim _{n \rightarrow \infty} c_{i_{0}}^{(n)}=y_{0}\left(i_{0}\right)>0$. For a sufficiently large $l, c_{i_{0}}^{\left(n_{i_{l}}\right)}>0$. It follows that, for a sufficiently large $l$,

$$
\begin{aligned}
1 & =a_{i_{0}}^{\left(n_{i_{l}}\right)} c_{i_{0}}^{\left(n_{i_{l}}\right)}+\sum_{j \neq i_{0}}^{\infty} a_{j}^{\left(n_{i_{l}}\right)} c_{j}^{\left(n_{i_{l}}\right)} \\
& \leq\left|a_{i_{0}}^{\left(n_{i_{l}}\right)}\right| c_{i_{0}}^{\left(n_{i_{l}}\right)}+\sum_{j \neq i_{0}}^{\infty}\left|a_{j}^{\left(n_{i_{l}}\right)}\right|\left|c_{j}^{\left(n_{i_{l}}\right)}\right| \\
& \leq \sum_{j=1}^{\infty}\left|c_{j}^{\left(n_{i_{l}}\right)}\right|=1 .
\end{aligned}
$$

Thus $1=a_{i_{0}}^{\left(n_{i_{l}}\right)}=\left|a_{i_{0}}^{\left(n_{i_{l}}\right)}\right|$ for a sufficiently large $l$, so $r=1$. Therefore $\lim _{n \rightarrow \infty} a_{i_{0}}^{(n)}=$ 1. We claim that $\lim _{n \rightarrow \infty} a_{j}^{(n)}=a_{j}$ for all $j \in \mathbb{N} \backslash J$. Let $j_{0} \in \mathbb{N} \backslash J$ be fixed. Let $\left\{a_{j_{0}}^{\left(n_{i}\right)}\right\}$ be any subsequence of $\left\{a_{j_{0}}^{(n)}\right\}$. As the set $\left\{a_{j_{0}}^{\left(n_{i}\right)}\right\}$ is a bounded subset of $\mathbb{C}$, there exist a subsequence $\left\{a_{j_{0}}^{\left(n_{i_{l}}\right)}\right\}$ and a complex number $\rho$ with $|\rho| \leq 1$ such that $\lim _{l \rightarrow \infty} a_{j_{0}}^{\left(n_{i_{l}}\right)}=\rho$. Since

$$
1=\lim _{n \rightarrow \infty}\left|x_{n}^{*}\left(h\left(x_{n}\right)\right)\right| \leq \lim _{n \rightarrow \infty}\left|f\left(x_{n}\right)\right|\left|x_{n}^{*}\left(z_{0}\right)\right| \leq 1,
$$

we have

$$
1=\lim _{n \rightarrow \infty}\left|x_{n}^{*}\left(z_{0}\right)\right|=\lim _{n \rightarrow \infty}\left|\sum_{j=1}^{\infty} a_{j}^{(n)} \lambda_{j} b_{j}\right| .
$$

It follows that for fixed $i_{0} \in J$,

$$
\begin{aligned}
1 & =\lim _{l \rightarrow \infty}\left|\sum_{j=1}^{\infty} a_{j}^{\left(n_{i_{l}}\right)} \lambda_{j} b_{j}\right| \\
& \leq \lim _{l \rightarrow \infty}\left|a_{j_{0}}^{\left(n_{i_{l}}\right)} \lambda_{j_{0}} b_{j_{0}}+a_{i_{0}}^{\left(n_{i_{l}}\right)} b_{i_{0}}\right|+\sum_{j \in \mathbb{N} \backslash\left\{i_{0}, j_{0}\right\}} b_{j} \\
& \leq b_{j_{0}}+b_{i_{0}}+\sum_{j \in \mathbb{N} \backslash\left\{i_{0}, j_{0}\right\}} b_{j}=1 .
\end{aligned}
$$


Thus

$$
\lim _{l \rightarrow \infty}\left|a_{j_{0}}^{\left(n_{i_{l}}\right)} \lambda_{j_{0}} b_{j_{0}}+a_{i_{0}}^{\left(n_{i_{l}}\right)} b_{i_{0}}\right|=\left|\rho \lambda_{j_{0}} b_{j_{0}}+b_{i_{0}}\right|=b_{j_{0}}+b_{i_{0}}
$$

showing $\rho \lambda_{j_{0}}=1$. We have $\rho=a_{j_{0}}$. Therefore $\lim _{n \rightarrow \infty} a_{j_{0}}^{(n)}=a_{j_{0}}$. Since the $\mathrm{w}^{*}$ topology in $l_{\infty}$ is the convergence in each coordinate, we complete the proof.

By Theorem 2.3 in [3], $\Pi\left(l_{p}\right)$ is the numerical Šilov boundary for $\mathcal{A}_{\infty}\left(B_{l_{p}}: l_{p}\right)$ for each $1<p<\infty$. The following is an application of Theorem 2.2 to the numerical Šilov boundary.

Theorem 2.3. $S:=\left\{\left(x, x^{*}\right) \in \Pi\left(l_{1}\right):\left|x^{*}\left(e_{n}\right)\right|=1\right.$ for all $\left.n \in \mathbb{N}\right\}$ is the numerical Silov boundary for $\mathcal{A}_{\infty}\left(B_{l_{1}}: l_{1}\right)$.

Proof. It is easy to show that $S$ is $\left(\|\| \times w^{*}\right)$-closed. By Theorem 2.2 and the fact that every $\left(\|\| \times w^{*}\right)$-closed numerical boundary for $\mathcal{A}_{\infty}\left(B_{l_{1}}: l_{1}\right)$ contains all numerical strong-peak points, it suffices to prove that $S$ is a numerical boundary for $\mathcal{A}_{\infty}\left(B_{l_{1}}: l_{1}\right)$. Let $h \in \mathcal{A}_{\infty}\left(B_{l_{1}}: l_{1}\right)$ and let $\epsilon>0$. Choose $\left(x_{0}, x_{0}^{*}\right) \in \Pi\left(l_{1}\right)$ such that $v(h)-\epsilon<\left|x_{0}^{*}\left(h\left(x_{0}\right)\right)\right|$. Let $x_{0}:=\left(v_{n}\right)_{n}, x_{0}^{*}:=\left(w_{n}\right)_{n}$ and $h:=\left(h_{n}\right)_{n}$ for some $h_{n} \in \mathcal{A}_{\infty}\left(B_{l_{1}}\right)$. We claim that there exists a complex sequence $\left\{\lambda_{n}\right\}$ in the unit disk such that if

$$
z_{n}^{*}:=\sum_{1 \leq j \leq n} \lambda_{j} e_{j}+\sum_{j>n} w_{j} e_{j}
$$

for all $n \in \mathbb{N}$, then $\left(x_{0}, z_{n}^{*}\right) \in \Pi\left(l_{1}\right)$ and $\left|z_{n+1}^{*}\left(h\left(x_{0}\right)\right)\right| \geq\left|z_{n}^{*}\left(h\left(x_{0}\right)\right)\right|>v(h)-\epsilon$ for all $n \in \mathbb{N}$. If $\left|w_{1}\right|=1$, let $\lambda_{1}:=w_{1}$. Otherwise $w_{1}=0$; hence

$$
\left(x_{0}, \lambda e_{1}+\sum_{n>1} w_{n} e_{n}\right) \in \Pi\left(l_{1}\right) \text { for all }|\lambda| \leq 1 .
$$

If $h_{1}\left(x_{0}\right)=0$, let $\lambda_{1}:=1$. Assume that $h_{1}\left(x_{0}\right) \neq 0$. We define a nonconstant 1-degree polynomial $\psi_{1}: \bar{D}(0,1) \rightarrow \mathbb{C}$ by

$$
\psi_{1}(\lambda):=\lambda h_{1}\left(x_{0}\right)+\sum_{n>1} w_{n} h_{n}\left(x_{0}\right)(|\lambda| \leq 1) .
$$

Since $\max _{|\lambda|=1}\left|\psi_{1}(\lambda)\right| \geq\left|\psi_{1}\left(w_{1}\right)\right|=\left|x_{0}^{*}\left(h\left(x_{0}\right)\right)\right|$, by the Maximum Modulus Theorem, there exists a complex number $\lambda_{1}$ with $\left|\lambda_{1}\right|=1$ such that

$$
\left|\psi_{1}\left(\lambda_{1}\right)\right| \geq\left|x_{0}^{*}\left(h\left(x_{0}\right)\right)\right|>v(h)-\epsilon .
$$

Let $z_{1}^{*}:=\lambda_{1} e_{1}+\sum_{n>1} w_{n} e_{n}$. Then $\left(x_{0}, z_{1}^{*}\right) \in \Pi\left(l_{1}\right)$ and $\left|z_{1}^{*}\left(h\left(x_{0}\right)\right)\right| \geq\left|x_{0}^{*}\left(h\left(x_{0}\right)\right)\right|>$ $v(h)-\epsilon$. If $\left|w_{2}\right|=1$, let $\lambda_{2}:=w_{2}$. Otherwise $w_{2}=0$; hence

$$
\left(x_{0}, \lambda_{1} e_{1}+\lambda e_{2}+\sum_{n>2} w_{n} e_{n}\right) \in \Pi\left(l_{1}\right) \text { for all }|\lambda| \leq 1 .
$$

If $h_{2}\left(x_{0}\right)=0$, let $\lambda_{2}:=1$. Assume that $h_{2}\left(x_{0}\right) \neq 0$. We define a nonconstant 1-degree polynomial $\psi_{2}: \bar{D}(0,1) \rightarrow \mathbb{C}$ by

$$
\psi_{2}(\lambda):=\lambda_{1} h_{1}\left(x_{0}\right)+\lambda h_{2}\left(x_{0}\right)+\sum_{n>2} w_{n} h_{n}\left(x_{0}\right)(|\lambda| \leq 1) .
$$

Since $\max _{|\lambda|=1}\left|\psi_{2}(\lambda)\right| \geq\left|\psi_{2}\left(b_{2}\right)\right|=\left|\psi_{1}\left(\lambda_{1}\right)\right|>v\left(h_{0}\right)-\epsilon$, by the Maximum Modulus Theorem, there exists a complex number $\lambda_{2}$ with $\left|\lambda_{2}\right|=1$ such that

$$
\left|\psi_{2}\left(\lambda_{2}\right)\right| \geq\left|\psi_{1}\left(\lambda_{1}\right)\right|>v(h)-\epsilon .
$$

Let $z_{2}^{*}:=\sum_{1 \leq j \leq 2} \lambda_{j} e_{j}+\sum_{n>2} w_{n} e_{n}$. Then $\left(x_{0}, z_{2}^{*}\right) \in \Pi\left(l_{1}\right)$ and $\left|z_{2}^{*}\left(h\left(x_{0}\right)\right)\right| \geq$ $\left|z_{1}^{*}\left(h\left(x_{0}\right)\right)\right|>v(h)-\epsilon$. Continuing this process, we can get a complex sequence 
$\left\{\lambda_{n}\right\}$ in the unit disk satisfying the claim. Let $z^{*}:=\left(\lambda_{n}\right)_{n=1}^{\infty} \in l_{\infty}$. We will show that $\left(x_{0}, z^{*}\right) \in S$. Indeed, it follows that for each $n \in \mathbb{N}$,

$$
\left|z^{*}\left(x_{0}\right)-1\right|=\left|z^{*}\left(x_{0}\right)-z_{n}^{*}\left(x_{0}\right)\right| \leq \sum_{j>n}\left|\lambda_{j}-w_{j}\right|\left|v_{j}\right| \leq 2 \sum_{j>n}\left|v_{j}\right| \rightarrow 0,
$$

as $n \rightarrow \infty$. Thus $z^{*}\left(x_{0}\right)=1$. We will show that

$$
\lim _{n \rightarrow \infty}\left|z_{n}^{*}\left(h\left(x_{0}\right)\right)\right|=\left|z^{*}\left(h\left(x_{0}\right)\right)\right| .
$$

Let $h\left(x_{0}\right):=\left(\beta_{n}\right)_{n=1}^{\infty} \in l_{1}$. It follows that

$$
\left|z^{*}\left(h\left(x_{0}\right)\right)-z_{n}^{*}\left(h\left(x_{0}\right)\right)\right| \leq \sum_{j>n}\left|\lambda_{j}-w_{j}\right|\left|\beta_{j}\right| \leq 2 \sum_{j>n}\left|\beta_{j}\right| \rightarrow 0,
$$

as $n \rightarrow \infty$. Thus we have

$$
v(h)-\epsilon<\limsup _{n \rightarrow \infty}\left|z_{n}^{*}\left(h\left(x_{0}\right)\right)\right|=\left|z^{*}\left(h\left(x_{0}\right)\right)\right| \leq \sup _{\left(x, x^{*}\right) \in S}\left|x^{*}(h(x))\right| \leq v(h),
$$

which shows that $\sup _{\left(x, x^{*}\right) \in S}\left|x^{*}(h(x))\right|=v(h)$. Since $h \in \mathcal{A}_{\infty}\left(B_{l_{1}}: l_{1}\right)$ is arbitrary, we complete the proof.

\section{NUMERICAL PEAK POINTS}

\section{AND NUMERICAL STRONG-PEAK POINTS ON $C(K)$}

Theorem 3.1. Let $K$ be a compact Hausdorff topological space with at least two points. If $\left(x_{0}, x_{0}^{*}\right) \in \Pi(C(K))$ is a numerical peak point for $\mathcal{A}_{\infty}\left(B_{C(K)}: C(K)\right)$, then:

(a) There exists a unique $t_{0} \in K$ such that

$$
\left|x_{0}(t)\right|=1, \forall t \in K \text { and } x_{0}^{*}=\operatorname{sign}\left(x_{0}\left(t_{0}\right)\right) \delta_{t_{0}} .
$$

Hence $x_{0}$ is an extreme point of $B_{C(K)}$.

(b) $x_{0}$ is a peak point for $\mathcal{A}_{\infty}\left(B_{C(K)}\right)$.

Proof. Let $h \in S_{\mathcal{A}_{\infty}\left(B_{C(K)}: C(K)\right)}$ peak numerically at $\left(x_{0}, x_{0}^{*}\right)$. Then $v(h)$ $=\left|x_{0}^{*}\left(h\left(x_{0}\right)\right)\right|$. By Theorem 2.7 in [2], $1=v(h)=\|h\|$. Choose an element $t_{0} \in K$ such that $1=\left\|h\left(x_{0}\right)\right\|=\left|\delta_{t_{0}} \circ h\left(x_{0}\right)\right|=\left\|\delta_{t_{0}} \circ h\right\|$. We claim that $\left|x_{0}(t)\right|=1, \forall t \in K$. For every complex number $\lambda$ in the unit disk, the function $x_{0}+\lambda\left(1-\left|x_{0}\right|\right) \in C(K)$, and for every $t \in K$, it is satisfied that $\left|x_{0}(t)+\lambda\left(1-\left|x_{0}(t)\right|\right)\right| \leq 1$. Define the continuous function $\phi: \bar{D}(0,1) \rightarrow \mathbb{C}$ by

$$
\phi(\lambda):=\delta_{t_{0}}\left(h\left(x_{0}+\lambda\left(1-\left|x_{0}\right|\right)\right)\right)(|\lambda| \leq 1) .
$$

Note that $\phi$ is holomorphic on $D(0,1)$ and $|\phi(\lambda)| \leq 1$ for every $\lambda$ in the unit disk. Also $|\phi(0)|=\left|\delta_{t_{0}} \circ h\left(x_{0}\right)\right|=1$. Since $\phi$ attains its maximum modulus at $0, \phi$ is constant. We choose a complex number $\lambda_{0}$ satisfying the facts that $\left|\lambda_{0}\right|=1$ and $\left|x_{0}\left(t_{0}\right)+\lambda_{0}\left(1-\left|x_{0}\left(t_{0}\right)\right|\right)\right|=1$. So $\phi(0)=\phi\left(\lambda_{0}\right)$. The element $z_{0}:=x_{0}+\lambda_{0}\left(1-\left|x_{0}\right|\right)$ is in the unit ball of $C(K)$ and $\left|z_{0}\left(t_{0}\right)\right|=1$. Then $\left(z_{0}, \overline{z_{0}\left(t_{0}\right)} \delta_{t_{0}}\right) \in \Pi(C(K))$. Since $\left|\phi\left(\lambda_{0}\right)\right|=\left|\overline{z_{0}\left(t_{0}\right)} \delta_{t_{0}}\left(h\left(z_{0}\right)\right)\right|=v(h)=1$, we have $z_{0}=x_{0}$ and $x_{0}^{*}=\overline{x_{0}\left(t_{0}\right)} \delta_{t_{0}}$. Thus $\lambda_{0}\left(1-\left|x_{0}(t)\right|\right)=0$ for all $t \in K$, so $\left|x_{0}(t)\right|=1$ for all $t \in K$. So $x_{0}^{*}=\operatorname{sign}\left(x_{0}\left(t_{0}\right)\right) \delta_{t_{0}}$. The uniqueness of $t_{0}$ follows from Urysohn's Lemma. Therefore we have proved assertion (a). 
We will show that $\delta_{t_{0}} \circ h$ peaks at $x_{0}$. Let $y \in S_{C(K)}$ such that $1=\left|\delta_{t_{0}} \circ h(y)\right|=$ $\left\|\delta_{t_{0}} \circ h\right\|$. By the same argument as in the proof of assertion (a), we have $\left|y\left(t_{0}\right)\right|=1$. Note that $\left(y, \operatorname{sign}\left(y\left(t_{0}\right)\right) \delta_{t_{0}}\right) \in \Pi(C(K))$. Since

$$
1=\left|\operatorname{sign}\left(y\left(t_{0}\right)\right) \delta_{t_{0}}(h(y))\right|=\left\|\delta_{t_{0}} \circ h\right\|=\|h\|=v(h),
$$

we have $y=x_{0}$ and $x_{0}^{*}=\operatorname{sign}\left(y\left(t_{0}\right)\right) \delta_{t_{0}}$, which show assertion (b).

Theorem 3.2. (1) Let $K$ be a compact metrizable space.

Then $M:=\left\{\left(x, \operatorname{sign}(x(t)) \delta_{t}\right): x \in \operatorname{ext} B_{C(K)}, t \in K\right\}$ is the set of all numerical peak points for the space of 1-degree polynomials in $\mathcal{A}_{\infty}\left(B_{C(K)}: C(K)\right)$.

(2) If $K$ is any infinite compact topological space, then there are no numerical strong-peak points for $\mathcal{A}_{\infty}\left(B_{C(K)}: C(K)\right)$.

Proof. (1): By (a) of Theorem 3.1, it suffices to show that if $\left(x_{0}, \operatorname{sign}\left(x_{0}\left(t_{0}\right)\right) \delta_{t_{0}}\right) \in$ $M$, then it is a numerical peak point for the space of 1-degree polynomials in $\mathcal{A}_{\infty}\left(B_{C(K)}: C(K)\right)$. Since the subset of peak points in $S_{C(K)}$ for $\mathcal{A}_{\infty}\left(B_{C(K)}\right)$ is invariant under surjective linear isometries on $C(K)$, we can assume that $x_{0}(t)=1$ for all $t \in K$. Since $K$ is a metrizable space, there exists a dense subset $\left\{l_{n}\right\}$ in $K$. Choose $\left(\alpha_{n}\right) \in S_{l_{1}}$ with $\alpha_{n}>0$ for all $n \in \mathbb{N}$. We claim that there exists a function $y_{0} \in S_{C(K)}$ such that $y_{0}\left(t_{0}\right)=1$ and $0 \leq y_{0}(t)<1$ for all $t \in K \backslash\left\{t_{0}\right\}$. Indeed, let $d$ be a metric in $K$. Let

$$
A_{n}:=\left\{t \in K: d\left(t, t_{0}\right) \geq \frac{1}{n}\right\}(n \in \mathbb{N}) .
$$

Clearly $A_{n}$ is a closed subset of $K$ with $t_{0} \notin A_{n}$ for all $n \in \mathbb{N}$. By the Tietze Extension Theorem, for each $n \in \mathbb{N}$, there exists a sequence $\left\{z_{n}\right\}$ in $C(K)$ such that $0 \leq z_{n} \leq 1, z_{n}\left(t_{0}\right)=1$ and $z_{n}\left(A_{n}\right)=\{0\}$. We define the function

$$
y_{0}(t):=\sum_{n=1}^{\infty} \frac{1}{2^{n}} z_{n}(t)(t \in K) .
$$

We define a 1-degree polynomial $h \in \mathcal{A}_{\infty}\left(B_{C(K)}: C(K)\right)$ by

$$
h(x):=\sum_{n=1}^{\infty} \alpha_{n}\left(1+x\left(l_{n}\right)\right) y_{0}(x \in C(K)) .
$$

We claim that $h$ peaks numerically at $\left(x_{0}, \delta_{t_{0}}\right)$. Let $\left(z_{0}, z_{0}^{*}\right) \in \Pi(C(K))$ be such that $v(h)=\left|z_{0}^{*}\left(h\left(z_{0}\right)\right)\right|$. Since $2 \geq\|h\| \geq v(h) \geq \delta_{t_{0}}\left(h\left(x_{0}\right)\right)=2$, we have $2=v(h)=\|h\|$. Since $2=\left|z_{0}^{*}\left(h\left(z_{0}\right)\right)\right|=\left\|h\left(z_{0}\right)\right\|$, we have $z_{0}\left(l_{n}\right)=1$ for all $n \in \mathbb{N}$. Since $\left\{l_{n}\right\}$ is a dense subset of $K, z_{0}(t)=1=x_{0}(t)$ for all $t \in K$. Thus $z_{0}=x_{0}$. By the Riesz Representation Theorem on $C(K)^{*}$, there exists a unique regular complex Baire measure $\mu=v+i w$ on $K$ ( $v$ and $w$ are positive measures) satisfying

$$
z_{0}^{*}(x)=\int_{K} x(t) d \mu=\int_{K} x(t) d v+i \int_{K} x(t) d w(x \in C(K))
$$

with $\left\|z_{0}^{*}\right\|=|\mu|=1$. Since

$$
1=z_{0}^{*}\left(x_{0}\right)=\int_{K} x_{0} d v+i \int_{K} x_{0} d w=v(K)+i w(K),
$$

$v(K)=1, w(K)=0$. Thus $w=0$ and

$$
z_{0}^{*}(x)=\int_{K} x(t) d v(x \in C(K)) .
$$


It follows that

$$
2=\left|z_{0}^{*}\left(h\left(x_{0}\right)\right)\right|=\left|\int_{K} h\left(x_{0}\right) d v\right|=2\left|\int_{K} y_{0}(t) d v\right| \leq 2 \int_{K}\left|y_{0}(t)\right| d v \leq 2 .
$$

Thus $1=\int_{K}\left|y_{0}(t)\right| d v$. We claim that $v\left(\left\{t_{0}\right\}\right)=1$. Otherwise, by the regularity of $v$ and the choice of $y_{0}$, there exists an open subset $\theta_{0}$ of $K$ containing $t_{0}$ such that $v\left(K \backslash \theta_{0}\right)>0$. Let $\delta_{0}:=\max _{t \in K \backslash \theta_{0}}\left|y_{0}(t)\right|<1$. It follows that

$$
1=\int_{K}\left|y_{0}(t)\right| d v \leq v\left(\theta_{0}\right)+\delta_{0} v\left(K \backslash \theta_{0}\right)<v(K)=1,
$$

which is impossible. Thus $v\left(\left\{t_{0}\right\}\right)=1$ and $v\left(K \backslash\left\{t_{0}\right\}\right)=0$. Therefore, we have

$$
z_{0}^{*}(x)=\int_{K} x(t) d v=x\left(t_{0}\right) v\left(\left\{t_{0}\right\}\right)=x\left(t_{0}\right)=\delta_{t_{0}}(x)
$$

for all $x \in C(K)$, showing $z_{0}^{*}=\delta_{t_{0}}$.

(2): By (a) of Theorem 3.1, it suffices to show that if $\left(x_{0}, x_{0}^{*}\right) \in \Pi(C(K))$ with $x_{0} \in \operatorname{ext} B_{C(K)}$, then $\left(x_{0}, x_{0}^{*}\right)$ is not a numerical strong-peak point. Let $\left\{t_{n}\right\}$ in $K$ be the sequence such that there is a sequence $\left\{x_{n}\right\}$ in $B_{C(K)}$ such that $0 \leq x_{n} \leq$ $1, x_{n}\left(t_{n}\right)=1$, for all $n$ and $\operatorname{supp}\left(x_{n}\right) \cap \operatorname{supp}\left(x_{m}\right)=\emptyset(n \neq m)$. Let $h \in \mathcal{A}_{\infty}\left(B_{C(K)}\right.$ : $C(K))$ such that $1=v(h)=\left|x_{0}^{*}\left(h\left(x_{0}\right)\right)\right|$. We will show that $h$ cannot peak strongnumerically at $\left(x_{0}, x_{0}^{*}\right)$. By Theorem 2.7 in [2], we have $v(h)=\|h\|=1$. There exists a $t_{0} \in K$ such that $\operatorname{sign}\left(x_{0}\left(t_{0}\right)\right) \delta_{t_{0}} \circ h \in \mathcal{A}_{\infty}\left(B_{C(K)}\right)$ and

$$
1=\left\|h\left(x_{0}\right)\right\|=\left|\delta_{t_{0}}\left(h\left(x_{0}\right)\right)\right|=\left|\operatorname{sign}\left(x_{0}\left(t_{0}\right)\right) \delta_{t_{0}}\left(h\left(x_{0}\right)\right)\right|=\left\|\delta_{t_{0}} \circ h\right\| .
$$

Let $z_{n}:=x_{0}\left(1-x_{n}\right)$ for all $n \in \mathbb{N}$. Since the support of $x_{n}$ are pairwise disjoint, there is a positive integer $N$ such that $x_{n}\left(t_{0}\right)=0$ for all $n>N$. Let $\lambda_{n}:=$ $\operatorname{sign}\left(z_{n}\left(t_{0}\right)\right)$ for all $n>N$. Thus $\left(z_{n}, \lambda_{n} \delta_{t_{0}}\right) \in \Pi(C(K))$ for all $n>N$. Since $\left\{x_{n}\right\}$ is equivalent to a $c_{0}$-basis, then it converges weakly to 0 . By the Rainwater theorem, the sequence $\left\{z_{n}\right\}$ is in the unit ball of $C(K)$ and converges weakly to $x_{0}$. Since $C(K)$ has the Dunford-Pettis property, then it has also the polynomial DunfordPettis property [10, and so, if we follow the argument in the proof Proposition 4.1 in [1], then

It follows that

$$
\left|\lambda_{n} \delta_{t_{0}} \circ h\left(z_{n}\right)\right| \rightarrow 1
$$

$$
\left\|z_{n}-x_{0}\right\|=\left\|x_{0} x_{n}\right\| \geq\left|x_{0}\left(t_{n}\right) x_{n}\left(t_{n}\right)\right|=1
$$

for all $n \in \mathbb{N}$. Therefore, we have proved that $\left(x_{0}, x_{0}^{*}\right)$ is not a numerical strong-peak point.

It is known in 3], Theorem 5.2, that there is no numerical Šilov boundary for $\mathcal{A}_{\infty}\left(B_{C(K)}: C(K)\right)$ if $K$ is an infinite compact Hausdorff topological space.

Corollary 3.3. Let $n \in \mathbb{N}$. Then $M:=\left\{\left(x, \operatorname{sign}(x(t)) \delta_{t}\right) \in \Pi\left(l_{\infty}^{n}\right):|x(k)|=\right.$ 1 for all $k=1,2, \ldots, n$, for some $t=1,2, \ldots, n\}$ is the numerical Silov boundary for $\mathcal{A}_{\infty}\left(B_{l_{\infty}^{n}}: l_{\infty}^{n}\right)$.

Proof. $(\subseteq)$ : By Proposition 5.1 in 3], it follows.

$(\supseteq)$ : Clearly $M$ is $\left(\|\| \times w^{*}\right)$-closed. By Theorem $3.2(1), M$ is the set of all numerical peak points for $\mathcal{A}_{\infty}\left(B_{l_{\infty}^{n}}: l_{\infty}^{n}\right)$. Since $l_{\infty}^{n}$ is finite dimensional, $M$ is the set of all numerical strong-peak points. 


\section{REFERENCES}

1. M. D. Acosta, Boundaries for spaces of holomorphic functions on $C(K)$, Publ. Res. Inst. Math. Sci. 42 (2006), 27-44. MR2215434 (2007j:46073)

2. M. D. Acosta and S. G. Kim, Denseness of holomorphic functions attaining their numerical radii, Israel J. Math. 161 (2007), 373-386. MR.2350167

3. M. D. Acosta and S. G. Kim, Numerical boundaries for some classical Banach spaces, to appear in J. Math. Anal. Appl.

4. M. D. Acosta and M. L. Lourenco, Shilov boundary for holomorphic functions on some classical Banach spaces, Studia Math. 179 (2007), no. 1, 27-39. MR2291721

5. E. Bishop, A minimal boundary for function algebras, Pacific J. Math. 9 (1959), 629-642. MR0109305 (22:191)

6. F. F. Bonsall and J. Duncan, Numerical ranges II, London Math. Soc. Lecture Note Ser. 10 (Cambridge Univ. Press, 1973). MR0442682 (56:1063)

7. T. W. Gamelin, Uniform Algebra, Chelsea, New York, 1984.

8. J. Globevnik, Boundaries for polydisc algebras in infinite dimensions, Math. Proc. Cambridge Philos. Soc. 85 (1979), 291-303. MR516088 (80i:46046)

9. L. Harris, The numerical range of holomorphic functions in Banach spaces, Amer. J. Math. 93 (1971), 1005-1119. MR0301505 (46:663)

10. R. A. Ryan, Dunford-Pettis properties, Bull. Acad. Polon. Sci. Math. 27 (1979), 373-379. MR:557405 (80m:46018)

Department of Mathematics, Kyungpook National University, Daegu 702-701, South KOREA

E-mail address: sgk317@knu.ac.kr 